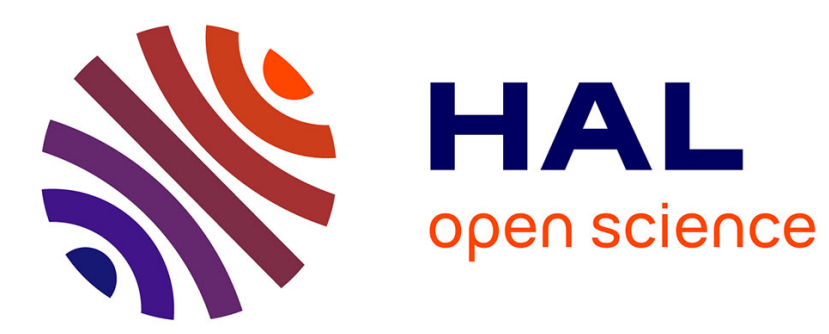

\title{
Construction du mouvement paysan et élaboration des politiques agricoles en Afrique subsaharienne
}

Denis Pesche

\section{To cite this version:}

Denis Pesche. Construction du mouvement paysan et élaboration des politiques agricoles en Afrique subsaharienne: Le cas du Sénégal. Politique africaine, 2009, 114 (2), 10.3917/polaf.114.0139 . hal01684414

\section{HAL Id: hal-01684414 https://hal.science/hal-01684414}

Submitted on 15 Jan 2018

HAL is a multi-disciplinary open access archive for the deposit and dissemination of scientific research documents, whether they are published or not. The documents may come from teaching and research institutions in France or abroad, or from public or private research centers.
L'archive ouverte pluridisciplinaire HAL, est destinée au dépôt et à la diffusion de documents scientifiques de niveau recherche, publiés ou non, émanant des établissements d'enseignement et de recherche français ou étrangers, des laboratoires publics ou privés. 


\section{CONSTRUCTION DU MOUVEMENT PAYSAN ET ÉLABORATION DES POLITIQUES AGRICOLES EN AFRIQUE SUBSAHARIENNE}

Le cas du Sénégal

Denis Pesche

Editions Karthala | «Politique africaine »

2009/2 $\mathrm{N}^{\circ} 114 \mid$ pages 139 à 155

ISSN 0244-7827

ISBN 9782811102364

Article disponible en ligne à l'adresse :

https://www.cairn.info/revue-politique-africaine-2009-2-page-139.htm

\section{Pour citer cet article :}

Denis Pesche, «Construction du mouvement paysan et élaboration des politiques agricoles en Afrique subsaharienne. Le cas du Sénégal », Politique africaine 2009/2 ( $\left.\mathrm{N}^{\circ} 114\right)$, p. 139-155.

DOI 10.3917/polaf.114.0139

Distribution électronique Cairn.info pour Editions Karthala.

(C) Editions Karthala. Tous droits réservés pour tous pays.

La reproduction ou représentation de cet article, notamment par photocopie, n'est autorisée que dans les limites des conditions générales d'utilisation du site ou, le cas échéant, des conditions générales de la licence souscrite par votre établissement. Toute autre reproduction ou représentation, en tout ou partie, sous quelque forme et de quelque manière que ce soit, est interdite sauf accord préalable et écrit de l'éditeur, en dehors des cas prévus par la législation en vigueur en France. Il est précisé que son stockage dans une base de données est également interdit. 


\title{
CONSTRUCTION DU MOUVEMENT PAYSAN ET ÉLABORATION

\author{
DES POLITIQUES AGRICOLES
}

\author{
EN AFRIQUE SUBSAHARIENNE \\ LE CAS DU SÉNÉGAL
}

\begin{abstract}
LE SÉNÉgal a PROMULGUÉ UNE LOI D'ORIENTATION AGRO-SYLVOPASTORALE EN 2004. AU COURS DU PROCESSUS D'ÉLABORATION DE CETTE LOI, LE CNCR, REPRÉSENTANT LES RURAUX SÉNÉGALAIS, A JOUÉ UN RÔLE CLÉ ET EST PARVENU À INFLUENCER SIGNIFICATIVEMENT LE TEXTE DE LOI, MALGRÉ UN CONTEXTE POLITIQUE QUI LUI ÉTAIT PLUTÔT hOSTILE. CET ARTICLE ANALYSE LES ÉTAPES ET LES CONDITIONS QUI ONT RENDU POSSIBLE CE TRAVAIL D'INFLUENCE TOUT EN SOULIGNANT LA FRAGILITÉ DES ACTEURS SOCIAUX EN PRÉSENCE ET DES COALITIONS QU'ILS FORMENT.
\end{abstract}

$\mathbf{E}_{n}$ juin 2004, le Sénégal adoptait la Loi d'orientation agro-sylvo-pastorale (Loasp) avec pour ambition de donner le cadre général des options de politique agricole et rurale pour les vingt prochaines années. Après plus d'une décennie de stratégies et de documents de politique agricole fortement influencés par la communauté des bailleurs de fonds, cette loi d'orientation apparaît comme résultant d'un processus original combinant initiative gouvernementale et participation significative de la société civile. Par sa durée, sa qualité et le nombre d'acteurs qui s'y sont impliqués, ce processus semble en effet se distinguer des injonctions de participation qui accompagnement maintenant systématiquement les phases d'élaboration de stratégies poli- tiques dans les pays en développement ${ }^{1}$. L'élaboration de la Loasp s'est échelonnée sur plus d'un an, depuis l'annonce officielle de son concept, en octobre 2002, une large consultation entre avril 2003 et mars 2004 puis sa promulgation en juin 2004. La Loasp est alors saluée par la presse et les observateurs comme un exemple de consensus entre le gouvernement et les ruraux sénégalais, représentés par le Conseil national de concertation et de coopération des ruraux (CNCR): cette loi constitue «une nouvelle vision à long terme qui découle d'un ensemble d'idées consensuelles capables d'insérer l'agriculture sénégalaise dans une dynamique de compétitivité et de durabilité ${ }^{2}$.

Pourtant, depuis l'alternance politique et 
l'arrivée d'Abdoulaye Wade à la présidence du pays (2000), le dialogue que les ruraux avaient réussi à instituer à la fin des années 1990 avec les pouvoirs publics avait été pratiquement rompu. Même si la tendance de fond du système politique sénégalais restait celle d'une scène politique compétitive, les années qui ont suivi l'alternance semblaient marquées par une tentation autoritaire et une recentralisation de l'État autour de la personne du Président ${ }^{3}$. Dans ce contexte politique plutôt défavorable pour le CNCR, quels sont les facteurs qui ont rendu possible ce compromis autour de la Loasp? Comment a pu se déployer l'influence du CNCR pour peser de façon significative sur le contenu de ce texte de politique générale?

Cet article se propose d'apporter des éléments de réponse à ces questions en mobilisant une analyse des ressorts du mouvement paysan, constitutifs de son influence ${ }^{4}$. Il s'agira de comprendre les interactions entre les quatre dimensions considérées comme les principaux facteurs explicatifs des dynamiques de mouvements sociaux: les structures d'opportunités politiques, les répertoires d'action collective, les mécanismes de mobilisation et les dynamiques plus cognitives de construction des identités et des représentations ${ }^{5}$.

Dans un premier temps, nous rappellerons à grands traits la genèse et le développement du mouvement paysan sénégalais avec en particulier la création en 1993 du Conseil national de concertation et de coopération des ruraux. Nous présenterons ensuite le cadre général et les grandes étapes du processus d'élaboration de la Loasp. Dans une troisième section, nous reviendrons sur quelques moments clés du rôle joué par le CNCR tout au long de ce processus analysant les modalités par lesquelles le CNCR a fait valoir son point de vue et l'a défendu. Enfin, la conclusion sera l'occasion de porter quelques interrogations sur les processus de construction de la représentation des intérêts dans les pays en développement.

\section{L'ÉMERGENCE D'UNE REPRÉSENTATION DES RURAUX SÉNÉGALAIS}

La compréhension du rôle joué par le CNCR dans le processus d'élaboration de la Loasp suppose de considérer cet événement comme une étape dans le processus de longue durée de construction de la représentation des intérêts des ruraux au Sénégal et des interactions entre le «mouvement paysan » sénégalais et l'État. Il est utile de rappeler à grands traits les principales étapes de cette construction pour mieux saisir les éléments constitutifs du mouvement paysan sénégalais. Le CNCR se crée en 1993 par la réunion de trois composantes $\mathrm{d}^{\prime}$ organisations rurales ${ }^{6}$ : les organisations coopératives, les plus anciennes mais fortement liées à l'État et très affaiblies depuis les politiques d'ajustement structurel; des fédérations nationales de groupements d'intérêts économiques (GIE), organisations économiques en général récentes et aux intérêts très morcelés et le mouvement associatif rural, représenté par la Fédération des organisations non gouvernementales du Sénégal (Fongs 7 ), qui a joué un rôle clé dans sa création.

La Fongs, créée en 1976, regroupe, contrairement à son appellation, des associations paysannes nées dans les années 1970 dans 
une logique d'autonomie par rapport aux interventions de l'État. Cette fédération s'élargit dans les années 1980 à plusieurs organisations paysannes de différentes régions du pays et renforce ses activités au début des années 1990 avec l'appui de plusieurs partenaires, coopérations bilatérales et ONG. Elle combine des activités d'appui à ses fédérations membres avec l'objectif de représenter leurs intérêts auprès des pouvoirs publics par la participation aux processus politiques concernant les ruraux. Au début des années 1990, elle cherche à participer à la négociation des politiques d'ajustement structurel pour le secteur agricole mais l'État ne lui reconnaît pas le statut de représentant des ruraux sénégalais. Les responsables de la Fongs amorcent alors la construction d'une large coalition des ruraux sénégalais qui prendra la forme du CNCR en 1993.

Le CNCR, après une période de consolidation (1993-1995) va s'imposer rapidement comme un interlocuteur incontournable auprès des pouvoirs publics et des bailleurs de fonds. Après avoir scellé une alliance avec les élus locaux, représentés par l'Association des présidents de communautés rurales. (APCR), il parvient à institutionnaliser un dialogue politique régulier avec les pouvoirs publics à partir de 1997 et participe activement aux négociations d'un important programme d'appui au secteur rural financé par la Banque mondiale ${ }^{8}$.

Une spécificité du CNCR est de se concentrer sur la fonction de représentation des intérêts des ruraux dans les processus politiques dans une attitude combinant un fort souci d'autonomie de pensée et une reconnaissance du rôle prééminent de
l'État dans ces processus. Cela se traduit par l'utilisation de répertoires d'action collective protestataire relativement mesurés, ne négligeant pas des épreuves de force comme le boycott des négociations avec l'État en $1996{ }^{9}$ mais aussi par des modes d'actions se rapprochant de ceux des groupes d'intérêts, avec par exemple la mobilisation d'une expertise proche des pouvoirs publics et l'utilisation des colloques et séminaires pour construire une vision et promouvoir des idées ${ }^{10}$. En affirmant l'importance d'engagements publics envers le monde rural et en exprimant clairement le «besoin d'État», le CNCR conforte en quelque sorte l'État face aux courants dominants portés par la communauté des bailleurs de fonds qui l'ont souvent critiqué et ont parfois cherché à le contourner ${ }^{11}$. En revendiquant, quelquefois avec vigueur, sa place dans le dialogue politique, le CNCR s'appuie sur le choix stratégique qu'ont fait les bailleurs de fonds d'impliquer les acteurs non étatiques dans les processus politiques et contribue ainsi à conforter cette option. Il y a donc un jeu à trois entre le CNCR, l'État et les bailleurs de fonds qu'il faut saisir pour comprendre la construction de l'influence politique des organisations paysannes sénégalaises.

Sans être en mesure d'apporter des données empiriques solides, on peut néanmoins souligner que le CNCR, et il l'admet souvent lui-même, connaît des faiblesses dans son fonctionnement interne et dans l'accomplissement de la mission de représentation des ruraux. Il est avéré qu'il regroupe des organisations couvrant la plupart des régions du pays mais avec, pendant longtemps, une sous-représentation 
de la région cotonnière et de la moyenne et haute vallée du fleuve Sénégal ${ }^{12}$. Par ailleurs, la grande hétérogénéité de ses fédérations membres et le nombre très réduit de salariés permanents à son siège rendent le fonctionnement interne difficile, avec une tendance à être en réaction face aux agendas extérieurs qui lui sont imposés plutôt que dans un travail de construction institutionnelle dans la durée.

Une spécificité importante du mouvement paysan sénégalais est de s'être impliqué, avec succès, dans la construction d'un réseau ouest-africain d'organisations paysannes. Préparée patiemment à l'occasion de plusieurs réunions internationales dans les années 1990, l'émergence du Réseau des organisations paysannes et de producteurs $\mathrm{d}^{\prime}$ Afrique de l'Ouest (Roppa ${ }^{13}$ ) en 2000 marque une étape importante dans les processus de représentation des intérêts des ruraux en Afrique de l'Ouest. Les responsables paysans sénégalais y ont joué sans conteste un rôle déterminant. La mise sur pied d'une structure de représentation régionale des agriculteurs est directement liée à la volonté de faire entendre la voix des ruraux suite à la constitution d'une Union douanière entre les pays $d^{\prime}$ Afrique de l'Ouest et l'élaboration d'une politique agricole de l'Union économique et monétaire ouest-africaine (UEMOA) en 2001.

Depuis sa création, le CNCR est porteur d'une volonté d'inscrire durablement sa vision du monde rural dans des politiques qu'il cherche à influencer, tant au niveau national qu'au niveau régional. Sans avoir revendiqué l'idée d'une loi d'orientation agricole en tant que telle, le mouvement paysan sénégalais a été régulièrement demandeur de la clarification des options politiques sénégalaises pour le monde rural et d'engagements dans la durée des pouvoirs publics. Le CNCR avait clairement exprimé, et régulièrement répété, son souhait de voir s'élaborer une loi-cadre pour l'agriculture et une loi foncière.

L'alternance politique de 2000 vient freiner l'essor du mouvement paysan sénégalais dans ses efforts d'institutionnalisation $\mathrm{du}$ dialogue politique sur les questions agricoles et rurales. Bien qu'ayant su préserver son autonomie vis-à-vis des pouvoirs publics, le CNCR est jugé trop proche du Parti socialiste par le nouveau pouvoir ${ }^{14}$. Le dialogue politique institué à la fin des années 1990 est rompu et ne reprendra que timidement en 2002, à l'occasion d'une crise de la filière arachidière qui s'est traduite par des situations d'impayé pour les producteurs : la Sonagraines ayant été privatisée en décembre 2001, la commercialisation de l'arachide s'est effectuée par le biais d'intermédiaires qui n'ont pas toujours tenu leurs engagements. En octobre 2002, le Président de la république annonce officiellement son projet de loi d'orientation agricole.

\section{GENÈsE et ÉLABORATION \\ DE LA LOI D'ORIENTATION AGRO-SYLVO-PASTORALE (LOASP)}

Le Sénégal, comme la plupart des pays $\mathrm{d}^{\prime}$ Afrique de l'Ouest, a connu l'ajustement structurel et la mise en place de politiques agricoles d'inspiration libérale depuis les années 1980. Le cadrage général des politiques s'appuie sur les documents de stratégie de réduction de la pauvreté, élaboré avec une forte implication des bailleurs 
143 Construction du mouvement paysan et élaboration des politiques agricoles...

de fonds ${ }^{15}$. Depuis la fin des années 1990, de nombreux documents officiels se sont succédé pour le monde rural et agricole: lettre de politiques diverses, documents stratégiques, souvent élaborés avec des consultants sans participation réelle des organisations paysannes. En 1998, le gouvernement sénégalais rend public un document d'orientation stratégique pour le secteur agricole et élabore, avec l'appui de l'Organisation des Nations unies pour l'alimentation et l'agriculture (FAO), un document provisoire de stratégie opérationnelle début 2000. L'alternance politique vient perturber ce processus et le ministère de l'Agriculture reprend le travail en proposant, fin 2001, une stratégie opérationnelle pour le secteur agricole ${ }^{16}$, mais les plans d'actions envisagés dans ce document sont restés pour partie lettre morte.

En 2002, dans un contexte de crise de la filière arachide et de fortes tensions en milieu rural, le Président et ses conseillers explorent l'idée d'une loi d'orientation agricole. Des responsables agricoles français et des conseillers politiques français alimentent cette réflexion et l'option de travailler sur un projet de loi d'orientation est prise par la présidence courant 2002. Le CNCR demandait depuis plusieurs années l'organisation d'un débat sur l'avenir du monde rural qui aurait pu déboucher sur des orientations claires pour les interventions publiques dans ce secteur. Informé $\mathrm{du}$ projet de loi d'orientation, le CNCR réclame, dans un mémorandum adressé en août 2002 au président de la République, «l'ouverture immédiate, sous l'autorité du Premier ministre, de négociations entre le gouvernement, le CNCR et les autres acteurs, sur la préparation de la loi d'orientation agricole ${ }^{17}$ ». La méthode adoptée par la présidence sera différente: un projet de loi est préparé dans le secret, par une équipe de quatre personnes ${ }^{18}$ et $l^{\prime}$ annonce officielle de la préparation d'une loi est faite par le président en octobre 2002. Une première mouture de la loi circule au sein de l'administration fin 2002 mais la première version publique ne sera diffusée que début avril 2003.

Dès la reprise du dialogue avec le gouvernement, le CNCR avait envisagé d'organiser un grand rassemblement pour démontrer sa capacité de mobilisation. Initialement prévu à l'automne 2002, il est repoussé début 2003 du fait du naufrage du Joola. Le 23 janvier 2003, le CNCR réussit finalement à mobiliser près de 30000 ruraux au stade Léopold Sédar Senghor de Dakar. Même si la manifestation est un demisuccès ${ }^{19}$, elle contribue cependant à conforter le poids politique du CNCR qui, à travers un «manifeste paysan » largement diffusé à l'occasion, insiste sur le besoin d'une loi d'orientation et d'une loi de réforme foncière spécifique. Pour le CNCR, de telles lois doivent résulter d'une large consultation sous forme « $\mathrm{d}^{\prime}$ assises nationales du monde rural pour définir une vision stratégique pour le monde rural ${ }^{20}$ ». Cette même période voit resurgir des fédérations paysannes nationales en sommeil depuis longtemps mais aussi la création de nouvelles fédérations revendiquant une représentativité des ruraux et questionnant la position hégémonique du CNCR dans ce domaine. Il s'agit en particulier de l'organisation «Forces paysannes», de l'Union nationale des Pêcheurs, Pasteurs et Paysans 
(3P) et du Mouvement sénégalais pour le développement. Le président de l'Union des 3P, Mouhamadou Madabo Dieng, membre du Parti démocratique sénégalais (PDS), s'exprime sans ambiguité: "Nous, nous sommes engagés à soutenir le président de la République durant son septennat, et nous l'avons dit clairement, mais cela ne signifie pas que notre organisation est une militante du PDS. Selon nos statuts, nous sommes d'ailleurs apolitiques ${ }^{21}$ ». Le président de Forces paysannes, Aliou Dia, auparavant affilié à l'Union pour le renouveau démocratique (URD), parti de Djibo Ka, a été investi par son mouvement pour les présidentielles de 2007 pour enfin se rallier à la candidature du président Wade. L'Union nationale des 3P s'est segmentée en trois organisations, portant à sept le nombre de «plateformes nationales». Sans disposer d'éléments empiriques beaucoup plus approfondis, on peut néanmoins affirmer, comme beaucoup d'observateurs du monde rural sénégalais, que l'émergence rapide et concomitante de ces organisations peut être interprétée comme la résultante d'initiatives visant à fragiliser la position $\mathrm{du}$ CNCR comme représentant unique des ruraux dans le dialogue avec l'État.

La première version de la loi est rendue publique le 2 avril 2003. Un court exposé des motifs est suivi de onze chapitres comportant au total quarante-cinq articles. Dans les grandes lignes, le projet de loi affirme de façon centrale la nécessité d'améliorer la compétitivité de l'agriculture sénégalaise pour qu'elle puisse rivaliser avec celle des autres pays sur les marchés internationaux. Le projet de loi spécifie l'existence de deux types d'exploitations: les exploitations familiales et les exploitations commerciales et industrielles ${ }^{22}$. Elle appelle à une réorganisation progressive de la production agricole, des filières et des marchés et contient un chapitre sur le foncier qui va susciter très vite de vives réactions d'acteurs diversifiés.

Les responsables du CNCR, des responsables politiques, des experts et même le ministère de l'Économie et des Finances émettent des doutes sur les propositions foncières inclues dans le projet de loi. Pour le CNCR, le projet de loi entérinerait une dualisation de l'agriculture sénégalaise et un risque majeur pour les exploitations familiales, très largement majoritaires: «il est hors de question que le régime foncier soit inclus dans le présent projet de loi » estime M. Cissokho, le président d'honneur du CNCR ${ }^{23}$.

Du côté des responsables politiques de l'opposition, les réactions sont aussi très vives. Les responsables socialistes reconnaissent que la Loi d'orientation agricole (LOA) est une chose appréciable mais mettent en garde contre ce qu'ils considèrent comme "une réforme foncière camouflée ${ }^{24}$ ». L'Alliance des forces du progrès $\left(\mathrm{AFP}^{25}\right)$ met en avant les risques que pourrait entraîner une réforme foncière hâtivement menée avec le choix de «la terre à ceux qui ont les moyens de l'exploiter ${ }^{26}$ ». En effet, le chapitre sur le foncier, le plus important du projet de loi (neuf articles et trois pages) avance explicitement l'idée d'une régulation foncière segmentée en deux marchés avec d'un côté une régulation assurée par les communautés rurales et, de l'autre côté, une gestion directe des attributions de terre par une agence placée sous 
145 Construction du mouvement paysan et élaboration des politiques agricoles...

la responsabilité de la présidence. «Cependant, ce nouveau Projet de loi va encore plus loin que le projet de provincialisation dans la réalisation de l'objectif du Président Wade de gérer personnellement le patrimoine foncier national, puisqu'il l'autorise à vendre les terres à des investisseurs privés sans l'aval d'aucune collectivité locale concernée et sans l'autorisation de l'Assemblée nationale ${ }^{27}$.» Ces tensions foncières sont particulièrement vives dans les zones périurbaines, dans la zone des Niayes et sur les terres irriguées de la vallée du fleuve. En définitive, face à la montée des mécontentements, largement relayés par la presse, le «volet foncier» est retiré de la loi par le gouvernement à la fin du mois d'avril.

Les autorités envisagent d'abord de mettre en débat le projet de loi dans un délai très court afin de finaliser les consultations pour le débat budgétaire 2004. Le CNCR réagit alors rapidement et se mobilise pour prendre une part active à la concertation. Il organise dès la mi-avril un atelier national d'information et de formation pour ses cadres nationaux et régionaux, et envisage une consultation nationale devant aboutir à un séminaire national fin mai avant d'aborder la phase de négociation proprement dite. Ces délais sont rapidement repoussés et le séminaire national se tiendra finalement mi-septembre 2003 à Dakar. Un rapport général (dix-huit pages) et une déclaration finale marquent alors la position du CNCR au moment de s'engager dans la négociation avec les pouvoirs publics.

Fin août 2003, ceux-ci mettent en place un comité national de finalisation de la loi $\mathrm{d}^{\prime}$ orientation agricole qui comprend douze membres issus de l'administration et des organisations paysannes nationales. Ce comité se réunira deux fois et validera le travail de rédaction d'une seconde version du projet de loi d'orientation, fin novembre 2003. Ce nouveau texte passera en conseil des ministres fin janvier 2004 et fera l'objet d'un débat parlementaire courant mars : il sera voté en mai puis promulgué en juin 2004.

\section{RETOUR SUR QUELQUES MOMENTS CLÉS DU PROCESSUS POLITIQUE ET ANALYSE dU RÔLE JOUÉ PAR LE CNCR}

Après cette rapide description du processus d'élaboration de la Loasp, il est utile de revenir sur certains aspects clés qui permettent d'éclairer les modalités de l'influence que le CNCR a pu avoir. La littérature sur les mouvements paysans s'inscrit dans celle plus large des mouvements sociaux ${ }^{28}$. Dans ce domaine, de récentes synthèses suggèrent de prendre en compte quatre principaux facteurs pour comprendre la dynamique de ces mouvements et leur capacité à faire entendre leur voix : les structures d'opportunités politiques, les répertoires d'action collective, les mécanismes de mobilisation et le travail de «cadrage» correspondant aux dynamiques cognitives de construction des identités et des représentations ${ }^{29}$. Le courant de l'analyse des mouvements sociaux s'est largement développé depuis les années 1970 avec, dans un premier temps, des tendances à privilégier l'un ou l'autre de ces facteurs. La tendance depuis les années 1990 semble être d'avoir une approche combinant, de façon pragmatique, ces différents facteurs 
et si possible dans une perspective dynamique pour saisir les processus de mobilisation et leurs interactions avec les processus politiques ${ }^{30}$.

Dans le cas présent, on peut mettre en avant les deux variables que sont le travail de cadrage et l'appréciation des opportunités politiques comme les deux principaux éléments pouvant permettre de mieux comprendre les ressorts de l'influence du CNCR sur l'élaboration de la Loasp. En effet, les répertoires d'action collective n'ont pas changé à l'occasion du processus d'élaboration de la Loasp: le CNCR a valorisé son expérience passée en combinant la réflexion et le débat interne, la mobilisation d'une expertise pointue et d'un réseau lui permettant de consolider des coalitions autour de ses positions avec une politique de large communication publique. Les mécanismes de mobilisation sont très liés au travail de cadrage et de concertation à travers les débats internes menés par le CNCR dans la première phase de consultation interne d'avril à septembre 2003.

\section{LE TRAVAIL DE CADRAGE :}

\section{LA CONSTRUCTION PROGRESSIVE}

\section{D'UNE VISION DE LA QUESTION PAYSANNE}

Une caractéristique particulière du CNCR est de s'être, dès sa création, engagé dans un travail de construction d'une vision paysanne de l'agriculture sénégalaise, dans une perspective de restaurer la fierté d'être paysan avec, de façon plus large, la revendication d'une pleine citoyenneté pour les ruraux, souvent plus mal dotée en investissements et services publics que les citadins. Le contexte même de la création du CNCR est l'organisation par la Fongs en janvier 1993 d'un forum sur "Quel avenir pour le paysan sénégalais? » où il s'agissait de s'interroger sur l'impact des politiques agricoles sur l'agriculture familiale et de réfléchir sur la capacité du mouvement paysan à influencer les politiques le concernant, et à mener des actions visant à l'amélioration des conditions de vie des paysans.

Ce processus de construction d'une vision a connu quelques étapes importantes avec un travail de réflexion entre 1997 et 1998 autour de la thématique: «Quelle place pour l'exploitation familiale dans une économie libéralisée ? ${ }^{31}$ » À travers ce document, les dirigeants paysans sénégalais espèrent que leur vision puisse « contribuer à la construction d'un consensus fort entre l'État, les cadres de ce pays, l'ensemble de nos concitoyens et nos partenaires au développement autour d'une politique en faveur de l'agriculture familiale». Le CNCR et la Fongs s'inscrivent alors d'emblée dans une optique de construire une vision d'avenir de l'agriculture qui puisse constituer le socle d'une politique nationale concertée.

Quels sont les points principaux de cette «vision paysanne»? Les organisations paysannes constatent que deux modes de production agricole coexistent au Sénégal: l'agriculture familiale et les entreprises agricoles à base de capitaux ${ }^{32}$. Malgré son caractère dominant en termes démographiques et de contribution au PIB agricole, l'agriculture familiale est souvent déconsidérée dans les politiques agricoles nationales au profit de l'agriculture à base de capitaux que les pouvoirs publics ont la tentation de privilégier dans les choix 
147 Construction du mouvement paysan et élaboration des politiques agricoles...

d'investissements et les mesures d'accompagnement. Le CNCR estime alors que l'État doit clarifier ses priorités et que l'agriculture familiale doit être privilégiée en zone pluviale, c' est-à-dire pour l'immense majorité du territoire. Le travail de réflexion conduit les responsables agricoles à formuler des propositions de politiques à court et moyen termes (en particulier en faveur d'un renforcement des capacités institutionnelles et des ressources humaines, $y$ compris d'ailleurs celles de l'État), mais aussi à long terme (foncier et formation agricole notamment).

Cette vision, portée par les dirigeants des organisations paysannes sénégalaises, intègre aussi la dimension internationale dans l'analyse spécifique des questions agricoles:

«La mondialisation et la libéralisation posent donc le problème de la coexistence de plusieurs modèles d'agriculture. Il serait vain de s'opposer au modèle agro-industriel. La plupart des pays développés et même les pays émergents comme ceux d'Amérique latine continueront à le développer. La question est de savoir si les accords internationaux permettront la survie et le développement $\mathrm{d}^{\prime}$ autres modèles et à quelles conditions $^{33}$.»

L'analyse des responsables paysans sénégalais prend acte de la dualisation de l'agriculture sénégalaise et, sans s'opposer à cet état de fait, insiste sur l'importance de mettre la priorité sur le soutien et la modernisation de l'agriculture familiale ${ }^{34}$, largement majoritaire dans le pays. Le CNCR insiste aussi sur la dimension politique de son projet en mettant en avant la citoyenneté et les revendications d'équité pour les ruraux en termes d'accès aux services et aux investissements publics.

Progressivement, les responsables paysans sénégalais, et plus largement ceux d'Afrique de l'Ouest vont développer cette vision et la «cristalliser» dans la notion d'exploitation familiale. L'adjectif agricole a disparu, soulignant ainsi la pluriactivité réelle des familles rurales. Le Roppa, créé en 2000, reprendra et affirmera cette vision $d^{\prime}$ une modernisation du secteur agricole et rural basée sur l'exploitation familiale. Son premier chantier de mobilisation sera l'influence sur la politique agricole l'UEMOA alors en gestation. La pression exercée par les organisations paysannes d'Afrique de l'Ouest se traduira par la prise en compte plus explicite de l'agriculture familiale dans les objectifs de cette politique ${ }^{35}$. Cette reconnaissance internationale contribuera à conforter le CNCR dans ses positions au niveau national. En effet, on doit replacer cette construction progressive de la vision des organisations paysannes sur l'avenir de l'agriculture dans le contexte d'une période de libéralisation des économies agricoles où l'influence croissante des institutions financières internationales se traduit par une certaine incohérence des mesures de politiques et l'absence d'une vision de long terme du fait d'une dégradation des capacités de l'État à élaborer des politiques ${ }^{36}$. L'investissement des organisations paysannes sur le niveau international leur permet, en retour, de se renforcer dans les arènes nationales, du fait de l'accroissement de leurs réseaux et de l'amélioration de leurs capacités d'analyse ${ }^{37}$. Cette insertion contribue aussi à 
façonner en partie la vision et les options du mouvement paysan par la mobilisation d'idées, de modes de raisonnements «importés » à l'occasion des rencontres et échanges internationaux ${ }^{38}$. Ainsi, le CNCR ne s'engage pas sans argument dans la consultation que lui propose le gouvernement autour de la Loasp à partir d'avril 2003. L'autre élément important dans la capacité d'influence du CNCR sur le processus d'élaboration de la Loasp aura été de formuler une contre-proposition. Plutôt que de faire des commentaires spécifiques sur tel ou tel aspect de la loi, le CNCR, après la phase de consultation de ses membres à la base, élaborera en octobre 2003 un contre-projet de loi, accentuant l'effet de cohérence de ses propositions fondées sur sa vision.

De la même façon, la force des positions du CNCR sur la question foncière a été confortée par les réactions multiformes de la société civile et politique mais aussi et surtout par l'argumentaire solide élaboré sur la base d'une réflexion conduite par l'organisation des ruraux sur la base de nombreux forums locaux depuis 2001. Ainsi, malgré le mode confidentiel d'élaboration de la première version de la LOA, le CNCR ne s'est pas retrouvé sans élément de référence pour soutenir ses réactions et élaborer les bases de ses contrepropositions, largement débattues au sein de ses organisations membres.

Enfin, un point essentiel dans ce processus de cadrage est le rôle joué par quelques leaders du CNCR et leurs conseillers, dont un en particulier, ancien directeur de l'Institut de recherche agricole du Sénégal, qui a accompagné et stimulé ce processus de réflexion interne et de construction d'une vision de la question paysanne au Sénégal. Sans tomber dans le piège de la personnification qui donnerait une importance accrue à quelques individus ${ }^{39}$, force est de constater que le rôle de médiateur, facteur clé dans les processus d'élaboration de politiques publiques, a été ici clairement assumé par le CNCR qui a su, par ses réseaux étendus au niveau de plusieurs sphères (organisations de producteurs, administration, bailleurs de fonds, champ politique, etc.) participer activement à la construction d'un compromis acceptable par les parties en présence.

\section{STRUCTURE DES OPPORTUNITÉS POLITIQUES, PERCEPTION}

DES MENACES ET DES OPPORTUNITÉS

La structure des opportunités politiques constitue un élément clé de l'analyse des mouvements sociaux. Elle forme la trame sur laquelle va se déployer la mobilisation et dont elle détermine, pour partie, l'éventail possible des résultats. Tilly et Tarrow identifient six propriétés d'un régime politique afin de caractériser sa structure des opportunités politiques ${ }^{40}$ : la multiplicité des centres indépendants de pouvoir, son ouverture envers de nouveaux acteurs, l'instabilité des alliances politiques, l'existence d'alliés influents et de supporter pour les challengers, l'attitude du régime face à la protestation collective et la mobilisation (recul) et des changements significatifs dans ces cinq éléments. La notion de structure des opportunités politiques doit néanmoins être utilisée avec précaution pour ne pas induire un schéma explicatif trop déterministe. McAdam, Tarrow et Tilly insistent 
149 Construction du mouvement paysan et élaboration des politiques agricoles...

sur l'importance de ne pas considérer la notion de structure des opportunités politiques comme une donnée objective expliquant de façon mécanique les processus de mobilisation mais d'observer la façon dont les acteurs interprètent les situations pour leur attribuer un caractère de menace ou $d^{\prime}$ opportunité ${ }^{41}$. Cette posture permet d'articuler la prise en compte d'éléments plus structurels avec l'interprétation que peuvent en faire les leaders des organisations dans leur travail de cadrage.

Dans le cas du Sénégal, les bailleurs de fonds sont des acteurs importants à prendre en compte dans l'appréciation des structures d'opportunités politiques. Depuis l'alternance, le gouvernement sénégalais, sous l'impulsion directe du chef de l'État, s'implique dans les débats sur les questions agricoles au niveau international et fait du secteur agricole une cible privilégiée de sa rhétorique sur le développement économique. L'affichage principalement international de ses ambitions dans le domaine, avec l'implication du président Wade dans le Nouveau partenariat pour le développement de l'Afrique (Nepad) et l'organisation du «Dakar agricole» en février 2005, ne trouve pas de traduction claire sur le plan national qui voit plutôt se succéder, y compris depuis la promulgation de la LOA, des plans annuels centrés sur un produit agricole phare. Juste après l'alternance, l'émergence d'un programme baptisé "Sénégal agricole», en mettant l'accent sur le soutien à l'exportation, tend à accentuer l'opposition entre les deux modèles d'agriculture déjà bien établie dans les esprits. Le trait commun des différentes initiatives gouvernementales dans le secteur agricole est sans doute leur volontarisme comme en témoigne l'intitulé de la dernière en date, la «Grande offensive agricole pour la nourriture et l'abondance» (2008).

L'Union européenne, chef de file des bailleurs de fonds au Sénégal pour le secteur rural, avait publiquement manifesté son désaccord en 2001 face à l'option du gouvernement sénégalais de mettre le secteur agricole au premier plan du document de stratégie de réduction de la pauvreté. La Banque mondiale est engagée depuis de nombreuses années au Sénégal dans plusieurs programmes d'investissement en milieu rural et de restructuration des services agricoles. Son dernier projet en date, le Programme des services agricoles et organisations de producteurs (Psaop), comporte un volet mis en œuvre par l'Association sénégalaise pour la promotion des petits projets de développement à la base (Asprodeb), structure d'exécution de projet pilotée par le CNCR et l'APCR. Depuis 2001, les bailleurs de fonds réclament du gouvernement des éclaircissements sur sa politique agricole et rurale. Comme nous l'avons vu plus haut, le CNCR a construit son poids politique en partie dans un dialogue, parfois tendu, entre à la fois le gouvernement et les bailleurs de fonds, en particulier la Banque mondiale.

L'alternance politique au Sénégal a été suivie d'une dégradation notoire du dialogue entre le CNCR et l'État et par l'émergence d'idées et d'images de politiques agricoles survalorisant le potentiel du secteur privé et des capitaux extérieurs à l'agriculture. L'affaiblissement du dialogue politique et l'accentuation d'une vision duale de l'agriculture, avec une préférence 
clairement exprimée par le nouveau gouvernement pour l'agriculture d'entreprise, suscite des réactions du CNCR et créé les conditions d'une mobilisation par l'accroissement du sentiment de menace, vécu en particulier autour des questions foncières. Ce contexte, renforcé par la crise arachidière, est propice à la remobilisation des ruraux, ce que saura exploiter le CNCR.

Dans ce contexte, les bailleurs de fonds ne sont pas forcément à l'unisson sur les questions de politique agricole. Cette structure «ouverte» se confirmera par la difficulté qu'ils auront à prendre une position sur le projet de loi en avril 2003. Leur désaccord tournait autour de la notion de souveraineté alimentaire, notion qui ouvre sur des débats plus larges mais rarement assumés publiquement, autour du degré de libéralisation économique et de protection de l'agriculture nationale. L'Union européenne et la FAO avaient aussi été très impliquées dans le processus de finalisation du «Document de stratégie opérationnelle» (2001) et voyaient mal l'utilité d'une loi d'orientation. Après l'envoi d'une lettre commune de commentaire, chaque bailleur de fonds a fait ses propres commentaires de façon officieuse au ministre de l'Agriculture. La France a joué un rôle particulier dans ce processus, en défendant la notion de souveraineté alimentaire et l'importance d'une concertation interprofessionnelle au sein des filières, options jugées trop interventionnistes par d'autres bailleurs ${ }^{42}$.

La question de l'influence française dans l'élaboration de la Loasp appelle à des réponses nuancées. D'un côté, le rôle de la France est manifeste, à la fois direct et indirect.
Il est direct sur le choix de l'instrument de politique publique que représente une loi d'orientation, à travers les conseillers français du Président; sur la concertation, fortement suggérée par des responsables d'organisations paysannes et agricoles françaises aux autorités sénégalaises et sur sa rédaction à laquelle ont participé quelques Français. La toute première version de la loi est rédigée par un Français, conseiller juridique de la présidence. En amont, plusieurs consultations d'experts agricoles français ont alimenté cette première rédaction. Cette version est ensuite reprise et amendée par une équipe de quatre personnes dont un conseiller technique français ${ }^{43}$, qui a travaillé en réseau avec le comité franco-sénégalais mis en place par le ministère français de l'Agriculture depuis fin 2002.

L'influence française est aussi indirecte par les références agricoles de quelques acteurs sénégalais impliqués dans le processus $^{44}$. Mais il serait sans doute erroné d'attribuer un rôle trop prééminent à la France car ce serait minimiser la volonté des acteurs sénégalais (la présidence comme le CNCR) de produire un cadre d'orientation pour l'agriculture sur le long terme. L'engagement dans la consultation, les nombreuses réactions qu'a suscité la première version de la loi démontrent ${ }^{45}$ à l'évidence le caractère authentique du débat autour des questions agricoles et l'engagement des acteurs concernés : si l'influence française a pu se déployer sur certains aspects techniques, ce processus est pleinement sénégalais dans ses dimensions politiques. Ceci d'autant plus que, pour la première fois, un document de stratégie concernant 
151 Construction du mouvement paysan et élaboration des politiques agricoles...

le secteur agricole a été mis en discussion au Parlement.

En définitive, le CNCR a su jouer de la réouverture du dialogue avec le gouvernement, même dans un contexte plutôt hostile, et de la faible cohésion du groupe des bailleurs de fonds, pour mettre en forme ses positions et les défendre de façon articulée sur la base de la contre proposition de loi élaborée fin septembre 2003. La proximité entre le CNCR et la coopération suisse a aussi facilité les consultations informelles entre l'organisation des ruraux et quelques bailleurs de fonds. Il faut aussi signaler le soutien indirect ou parfois direct qu'a obtenu le CNCR dans le processus de concertation lui-même par des engagements financiers de la Banque mondiale, des coopérations française et suisse pour le financement $d u$ processus en tant que tel ${ }^{46}$. En définitive, le processus de consultation peut être considéré comme relativement consistant en comparaison à des processus participatifs plus formels comme ceux accompagnant l'élaboration des cadres stratégiques de lutte contre la pauvreté ${ }^{47}$.

Comme précisé dans la section antérieure, le processus d'élaboration de la Loasp peut être divisé en deux moments: la consultation autour du premier projet de loi, avec une consultation interne au mouvement paysan animée par le CNCR (avril à septembre 2003) et la négociation proprement dite pour aboutir au second projet de loi qui sera ensuite examiné par le gouvernement et le Parlement (octobre 2003 à mars 2004). Dans la première phase, la consultation interne animée par le CNCR sera un moment fort pour consolider le poids politique de l'organisation en s'assurant d'un large soutien de sa base. Plus de trente ateliers locaux, onze ateliers régionaux permettront la participation directe de plus de 3000 producteurs aux débats. Le séminaire national de septembre 2003, en donnant l'occasion au CNCR de rendre publique ses analyses et propositions autour de la loi, le mettra en position de force pour négocier le nouveau texte de loi avec le gouvernement.

En définitive, le travail de cadrage du CNCR et sa capacité à saisir les opportunités politiques lui ont permis de jouer un rôle décisif dans l'élaboration de la Loasp. Il a pu faire valoir ses positions, soit directement, soit grâce à des coalitions d'acteurs plus ou moins formalisées. Le retrait de la question foncière, la limitation du rôle de l'État dans la régulation des filières et la place accordée aux organisations de producteurs, la reconnaissance de la coexistence de deux formes d'agriculture sont autant de points clés inscrits dans la nouvelle loi. Peut-on parler pour autant d'une victoire? Les observateurs ont évoqué à l'époque une loi «consensuelle».

Comment apprécier réellement l'évolution du contenu de la Loi au long du processus de concertation? Un travail d'analyse de contenu réalisé sur trois textes (la première version, le contre-projet du CNCR et la Loasp promulguée) montre que les principaux changements se sont traduits par une juxtaposition de finalités et d'objectifs parfois divergents plutôt que par des choix clairs. Les inflexions du texte ont plutôt porté sur les rôles respectifs des différents acteurs, en restreignant celui de l'État et en réaffirmant celui des organisations de producteurs et du secteur privé ${ }^{48}$. Si le 
texte final paraît consensuel, n'est-ce pas lié finalement au fait qu'il intègre les différents points de vue sans se prononcer réellement sur une orientation claire pour le long terme ni préciser les modalités opérationnelles qui permettraient véritablement de parler de politique publique, avec des instruments bien identifiés?

Une fois promulgué, le texte de loi a été traduit en cinquante-deux engagements devant être pris dans des délais différents (entre trois mois et vingt ans!). Sept groupes thématiques ont été constitués entre fin 2005 et courant 2006 pour la mise en œuvre de la loi. L'animation de ces groupes a été confiée à des institutions différentes ${ }^{49}$, sous la coordination du ministère de l'Agriculture à travers la Direction de l'analyse, de la prévision et des statistiques (Daps). En 2007, trois ans après la promulgation de cette Loi d'orientation agro-sylvo-pastorale, aucun décret $d$ 'application n'avait été signé: T. Niang analyse cette absence de mise en œuvre de la Loasp comme le résultat d'une absence de portage politique, de capacité de pilotage de l'application de la Loasp et d'engagements financiers adéquats ${ }^{50}$. On pourrait aussi rajouter le fait que le mouvement paysan sénégalais s'est trouvé fragilisé par l'apparition de nouvelles fédérations, le CNCR cherchant à maintenir son influence sur des dispositifs qu'il estime importants, comme le Programme de soutien aux services agricoles et aux organisations paysannes (PSAOP2) plutôt que d'investir ses ressources humaines limitées dans un processus politique incertain.

En effet, chaque année, le secteur agricole fait l'objet de programmes et de dispositifs fortement promus par la présidence de la République et dont les liens avec la Loasp sont ténus voire inexistants. C'est le cas par exemple du Plan Reva (Retour vers l'agriculture) qui cherche à accompagner l'installation de jeunes et migrants de retour et suscite de vives réactions des organisations de producteurs sur les incidences foncières éventuelles et la non-prise en compte des débouchés commerciaux. On constate donc un fort dualisme des politiques envers le secteur agricole, avec d'un côté un cadre de politique «consensuel», assez général, mais non mis en œuvre et, d'un autre côté, des initiatives très médiatisées politiquement.

Le processus d'élaboration de la Loasp a été l'occasion pour le CNCR de revenir en force sur la scène nationale de l'élaboration des politiques agricoles et rurales. Ce retour, en partie fondé sur sa capacité à produire une vision d'avenir de l'agriculture sénégalaise et à bâtir des coalitions d'acteurs pour la défendre, semble aujourd'hui menacé par l'inertie gouvernementale et la personnalisation croissante du processus politique autour du président de la République. L'émergence de fédérations paysannes revendiquant leur place dans la concertation avec les pouvoirs publics a aussi fragilisé le CNCR qui se trouve aujourd'hui obligé de composer avec d'autres organisations à l'assise locale encore faible et largement articulées au champ politique ${ }^{51}$.

À travers cet exemple, on a pu montrer l'importance déterminante de la construction sur le moyen terme d'un projet et d'une vision pour structurer la mobilisation paysanne et lui conférer du poids dans les processus politiques. On aura aussi 
153 Construction du mouvement paysan et élaboration des politiques agricoles...

souligné la fragilité des mouvements sociaux et leur réversibilité partielle, confirmant en cela, s'il en était encore besoin, la non-linéarité des dynamiques de mobilisation et de construction de la représentation des intérêts dans des espaces politiques ouverts et changeants.

Denis Pesche Cirad ES-Arena, Montpellier

1. T. Niang, «Étude d'un processus national de définition et de mise en œuvre d'une politique publique sectorielle: le cas de la Loi d'orientation agro-sylvo-pastorale (Loasp) du Sénégal » in Réseau Impact, Modalités de dialogue entre société civile et État pour l'élaboration, la mise en œuvre et le suivi des politiques publiques de réduction de la pauvreté et des inégalités, Paris, «document de travail», octobre 2007, p. 46.

\section{Le Matin, 26 mai 2004.}

3. T. Dahou et V. Foucher, «Le Sénégal, entre changement politique et révolution passive», Politique africaine, $\mathrm{n}^{\circ}$ 96, décembre 2004, p. 7 et 13.

4. Les analyses développées dans cet article s'appuient sur plusieurs sources. En premier lieu, le travail de deux étudiantes stagiaires encadrées par l'auteur, Anne Chaboussou et Magali Ruello, Étude d'un processus de concertation pour l'élaboration d'une politique publique: le cas de la Loi d'orientation agro-sylvo-pastorale (Loasp) sénégalaise, Montpellier, mémoire de diplôme d'ingénieur en agronomie tropicale du Centre national d'études agronomiques des régions chaudes (CNEARC), novembre 2006; l'évaluation d'une des associations membres du CNCR, D. Pesche et M. Ba, Évaluation de la Fongs, Thiès, Fongs, 2006 ; plusieurs expertises sur des projets impliquant le CNCR auxquelles a participé l'auteur, M. R. Mercoiret, D. Pesche, J.-F Le Coq et J. Diaz, Le Renforcement des capacités des organisations paysannes et rurales: enseignements de l'expérience de la Banque mondiale, Banque mondiale et Centre de coopération internationale en recherche agricole pour le développement (Cirad), 2004, et la familiarité que l'auteur a acquise avec le mouvement paysan sénégalais par un suivi régulier depuis 1992.

5. D. McAdam, S. Tarrow et C. Tilly, Dynamics of Contention, Cambridge, Cambridge University Press, 2001; C. Tilly et S. Tarrow, Contentious Politics, Boulder, Paradigm publishers, 2007.
6. Le CNCR regroupait 7 fédérations à sa création en 1993, et 22 aujourd'hui (<www.cncr.org $>$ ).

7. N. Mc Keon, M. Watts et W. Wolford, Peasant Associations in Theory and Practice, Genève, United Nations Research Institute for Social Development, 2004 ; J. Faye et O. Ndiaye, Formulation d'un projet d'appui institutionnel aux organisations paysannes du Sénégal, Dakar, CNCR, Cirad, Mission de coopération et d'action culturelle (MCAC), 1998, p. 49.

8. Il s'agit du Programme Services agricoles et organisations de producteurs (Pasop) dont la gestation durera quatre ans (1996-2000) et sera l'occasion d'une négociation approfondie entre l'État sénégalais, les organisations de producteurs, représentés par le CNCR, et la Banque mondiale. Au final, le CNCR se verra confier la responsabilité de la mise en œuvre d'une des composantes du projet, M.-R. Mercoiret et al., Le Renforcement des capacités... op. cit.

9. N. McKeon et al., Peasant Associations..., op. cit., p. 16. 10. M. Offerlé, Sociologie des groupes d'intérêts, Paris, Montchrestien, 1994 ; E. Grossman et S. Saurugger, Les Groupes d'intérêts: action collective et stratégies de représentation, Paris, Armand Colin, 2006.

11. N. McKeon et al., Peasant Associations..., op. cit., p. 48. 12. Voir J. Faye et O. Ndiaye, Formulation d'un projet..., op. cit.

13. Le Roppa réunit à sa création des organisations et plate-formes paysannes de dix pays d'Afrique de l'Ouest. Il est présidé depuis sa création par Ndiogou Fall, président de la Fongs jusqu'en 2007 (<www.roppa.info >). 14. Les difficultés que le CNCR a rencontrées pour parvenir à instituer un dialogue politique avec le gouvernement socialiste à partir de 1997 devraient en ellesmêmes suffire à invalider cette thèse, en général fondée sur le constat de l'engagement politique au PS de certains responsables du CNCR. De façon synthétique, les responsables des fédérations d'éleveurs et de femmes ont plutôt massivement «migré » du Parti socialiste (PS) vers le Parti démocratique sénégalais (PDS), quelques leaders importants du CNCR, plutôt dans la mouvance des associations paysannes, sont restés fidèles au PS. L'ancien président du CNCR, Mamadou Cissokho, a toujours cherché à préserver l'autonomie du mouvement, estimant que l'engagement politique de certains leaders ne devait pas engager le mouvement dans son ensemble. On peut retrouver là une configuration classique où un mouvement ou un groupe d'intérêt se ménage des marges de manœuvres et des leviers pour l'action en jouant sur la tension entre engagement et autonomie. Localement, les configurations politiques sont multiples et le cas du delta du fleuve le montre bien. Sur ce point, voir T. Dahou, Entre parenté et politique, 


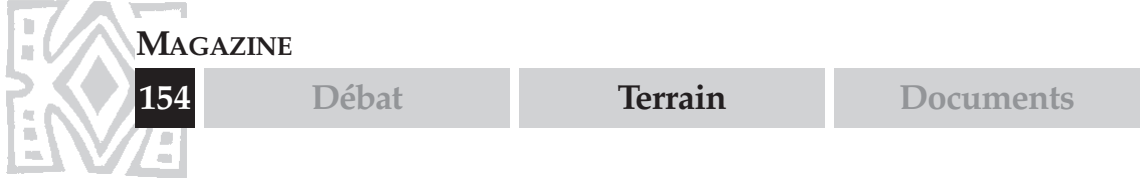

développement et clientélisme dans le Delta du Sénégal, Paris, Dakar, Karthala, Enda Graf Sahel, 2004, p. 85-102.

15. K. Cissokho et R. Touré, «Participation des acteurs sociaux et gouvernance d'État: le cas du cadre stratégique de lutte contre la pauvreté au Mali ", Politique africaine, $\mathrm{n}^{\circ} 99$, octobre 2005, p. 142-154. Sur l'uniformisation des processus de politiques agricole, voir aussi C. Oya, «From State dirigism to liberalisation in Senegal: four decades of agricultural policy shifts and continuities ", European Journal of Development Research, vol. $18, n^{\circ} 2$, 2006, p. 203-234.

16. République du Sénégal, ministère de l'Agriculture et de l'Élevage, Proposition de stratégie opérationnelle du secteur agricole, Rapport principal, Dakar, décembre 2001. 17. CNCR, Mémorandum du CNCR au Président de la République, août 2002, 3 p.

18. Cette équipe se compose de deux conseillers du Président (Omar Seck et Henri Savoie), d'un cadre du ministère de l'Agriculture (Mame Ndiobo Diene), et d'un de ses collaborateurs, un assistant technique français (Jean-René Cuzon).

19. Ses organisateurs avaient annoncé 100000 participants.

20. CNCR, Manifeste paysan du 26 janvier 2003.

21. Le Soleil, 30 janvier 2003.

22. Articles 12 et 13 du projet.

23. Compte rendu de l'atelier d'information et de formation des responsables locaux et régionaux du CNCR, Thiès, 12-13 avril 2003.

24. L'Actuel, $\mathrm{n}^{\circ} 460$, du 19 avril 2003.

25. L'AFP est un parti politique né d'une scission du PS en 1999: son leader, Moustapha Niasse est arrivé troisième aux élections présidentielles de 2000 avec $16,8 \%$ des voix. Il occupera pendant une courte période le poste de premier ministre d'Abdoulaye Wade dont il a contribué à la victoire en le ralliant entre les deux tours. Il rejoindra rapidement une opposition critique à l'égard de la politique du gouvernement.

26. L'Info7, $\mathrm{n}^{\circ}$ 1340, 5-6 avril 2003.

27. Commentaires d'Ibrahima Sène, département économique et social du comité central du Parti de l'indépendance et du travail (PIT), 28 avril 2003 (commentaires écrits, recueillis par l'équipe du ministère de l'Agriculture en charge de la préparation de la Loasp). Le PIT est un petit parti - un député en 2001 - qui faisait partie de la coalition soutenant Abdoulaye Wade en 2000 et a participé au gouvernement avant de rejoindre l'opposition moins d'un an après.

28. On trouvera une bonne synthèse de cette littérature et ses développements sur le cas des mouvements paysans des pays africains dans G. Lachenmann, «Civil society and social movements in Africa », in J.-P. Jacob et P. Lavigne-Delville, Les Associations paysannes en
Afrique: organisation et dynamique, Paris, Apad, Karthala, IUED, 1994, p. 61-95.

29. D. McAdam, S. Tarrow et C. Tilly, Dynamics of Contention, op. cit.; C. Tilly et S. Tarrow, Contentious Politics, op. cit.

30. D. McAdam, S. Tarrow et C. Tilly, Dynamics of Contention, op. cit., p. 43-50.

31. Fongs, "Quelle place pour l'exploitation familiale dans une économie libéralisée?», texte préparatoire préparé par la Fongs avec l'appui de Jacques Faye, séminaire de Mbour, 1998. Ce processus a été appuyé par la FAO et par le gouvernement sénégalais.

32. Fongs, "Quelle place pour l'exploitation... », ibid. 33. Ibid., p. 26-41.

34. La notion d'agriculture familiale regroupe une grande diversité de formes de production qui partagent toutes le fait d'avoir un mode familial d'organisation de la production, avec une dimension patrimoniale importante. C'est cette forme d'agriculture qui a dominé et domine encore dans la plupart des pays du monde, y compris en Europe.

35 . Acte additionnel $n^{\circ} 03 / 2001$ portant adoption de la politique agricole de l'UEMOA, <www.uemoa.int/ actes/2001/acte_additionnel_03_2001.htm>. Voir en particulier le préambule et l'article 8.

36. C. Oya, «From State dirigisme to liberalisation in Senegal...», art. cit., p. 221.

37. E. Grossman et S. Saurugger, Les Groupes d'intérêts..., op. cit., p. 10 et 110. Voir aussi des stratégies similaires exploitant le niveau international comme cible du lobbying mais aussi comme ressource pour conforter, en retour, son poids vis-à-vis des États dans le secteur du coton: D. Pesche et K. Nubukpo, «L'Afrique du coton à Cancún: les acteurs d'une négociation », Politique africaine, $\mathrm{n}^{\circ} 95$, octobre 2004, p. 158-168.

38. On pourrait faire une étude approfondie des étapes de cette internationalisation des mouvements paysans africains avec, dans un premier temps, l'insertion dans des réseaux d'ONG et de bailleurs de fonds (Six S) puis la diversification des contacts et des échanges vers des organisations et des experts agricoles d'autres pays du Sud (Brésil par exemple) et du Nord (France, Belgique, Canada, etc.) mais aussi directement auprès de représentants d'agences internationales ou d'instances intergouvernementales. Ces différentes inscriptions contribuent à façonner pour partie le travail de cadrage des leaders qui les font vivre.

39. T. Niang, "Étude d'un processus national...», art. cit., p. 40. L'auteur accorde une grande importance à deux personnes, le conseiller du CNCR (Jacques Faye) et le cadre du ministère de l'Agriculture (Mame Ndiobo Diene) qui a coordonné le processus de consultation. Il est évident que ces personnes ont joué un grand rôle 


\section{Politique africaine}

155 Construction du mouvement paysan et élaboration des politiques agricoles...

mais elles doivent cette capacité à leurs positions dans l'espace social, leur capital social et leurs dispositions acquises à travers leurs trajectoires plutôt qu'à une simple convergence de vue conjoncturelle sur les questions agricoles.

40. C. Tilly et S. Tarrow, Contentious Politics, op. cit.

41. D. McAdam, S. Tarrow et C. Tilly, Dynamics of Contention, op. cit., p. 45-46.

42. Entretiens réalisés en 2006 par A. Chaboussou et M. Ruello auprès de différents acteurs concernés (en particulier le secrétaire du groupe des bailleurs de fonds, un assistant technique de la coopération française). A. Chaboussou et M. Ruello, Étude d'un processus de concertation pour l'élaboration d'une politique publique: le cas de la Loi d'Orientation Agro-Sylvo-Pastorale (Loasp) sénégalaise, Montpellier, mémoire de diplôme d'ingénieur en agronomie tropicale du CNEARC, novembre 2006. 43. Voir supra, note 19.

44. Le coordonateur sénégalais du processus, cadre du ministère de l'Agriculture, avait fait sa thèse sur l'élaboration des politiques agricoles en France. Le conseiller du CNCR dans ce processus avait lui aussi de solides connaissances sur l'agriculture française et le rôle des lois d'orientation en France dans les années 1960-1962. 45. La première version de la LOA diffusée en avril 2003 a suscité les réactions de plus de cinquante services, organisations ou personnes, consignées dans un document de $280 \mathrm{p}$.

46. Le coût total du processus de concertation a été estimé à environ 100000 euros: T. Niang, «Étude d'un processus national...», art. cit., p. 41. Même si ce coût est probablement sous-estimé, le fait de l'expliciter permet utilement de souligner que les processus participatifs ont un coût, ce que ne prennent généralement pas en compte les décideurs politiques. On observera aussi que la France a soutenu à la fois la formulation de la première version de la loi soumise par la présidence puis la construction d'une contre-version et enfin l'accompagnement de la diffusion de la loi.

47. Voir en particulier l'exemple du Mali où les modes de consultations n'ont pas donné une place réelle à la société civile: $\mathrm{K}$. Cissokho et $\mathrm{R}$. Touré, «Participation des acteurs sociaux et gouvernance d'État: le cas du Cadre stratégique de lutte contre la pauvreté au Mali», Politique africaine, $\mathrm{n}^{\circ} 99$, octobre 2005, p. 142-154. Quelques indicateurs témoignent de la «consistance» du processus consultatif: le fait que le CNCR ait pu négocier le calendrier, le fait qu'il ait été partie prenante du comité chargé de faire la synthèse des textes, etc.

48. A. Chaboussou et M. Ruello, Étude d'un processus de concertation..., op. cit., p. 37-85.

49. Le CNCR s'est vu confier la responsabilité du groupe «métier » chargé d'examiner la mise en place d'un statut de l'exploitation familiale et d'une couverture sociale des métiers de l'agriculture et du rural.

50. T. Niang, "Étude d'un processus national...", art. cit., p. 43 .

51. Alors que le CNCR avait pu négocier un rôle conséquent, à travers l'Asprodeb, dans la mise en œuvre du PSAOP, rôle d'ailleurs jugé positif par les évaluations externes, il a dû se résoudre à devenir un bénéficiaire potentiel, au même titre que d'autres fédérations paysannes de création récente, dans la seconde phase de ce projet. 